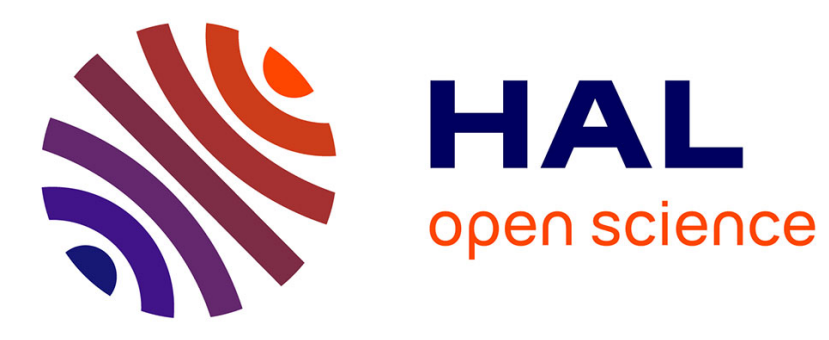

\title{
Sequencing and Welding of Molecular Single-Crystal Optical Waveguides
}

Luca Catalano, Julien Berthaud, Ghada Dushaq, Durga Prasad Karothu, Rachid Rezgui, Mahmoud Rasras, Sylvie Ferlay, Mir Wais Hosseini, Panče Naumov

\section{To cite this version:}

Luca Catalano, Julien Berthaud, Ghada Dushaq, Durga Prasad Karothu, Rachid Rezgui, et al.. Sequencing and Welding of Molecular Single-Crystal Optical Waveguides. Advanced Functional Materials, 2020, 30 (35), pp.2003443. 10.1002/adfm.202003443 . hal-03010611

\section{HAL Id: hal-03010611 https://hal.science/hal-03010611}

Submitted on 24 Nov 2020

HAL is a multi-disciplinary open access archive for the deposit and dissemination of scientific research documents, whether they are published or not. The documents may come from teaching and research institutions in France or abroad, or from public or private research centers.
L'archive ouverte pluridisciplinaire HAL, est destinée au dépôt et à la diffusion de documents scientifiques de niveau recherche, publiés ou non, émanant des établissements d'enseignement et de recherche français ou étrangers, des laboratoires publics ou privés. 


\section{WILEY-VCH}

\section{Sequencing and Welding of Molecular Single-Crystal Optical Waveguides}

Luca Catalano, Julien Berthaud, Ghada Dushaq, Durga Prasad Karothu, Rachid Rezgui, Mahmoud Rasras, Sylvie Ferlay, Mir Wais Hosseini, * Panče Naumov*

Dr. L. Catalano, Dr. G. Dushaq, Dr. D.P. Karothu, Dr. R. Rezgui, Prof. M. Rasras and Prof. P. Naumov

New York University Abu Dhabi, Abu Dhabi

129188, United Arab Emirates

E-mail: pance.naumov@nyu.edu

Dr. L. Catalano, J. Berthaud, Prof. S. Ferlay and Prof. M.W. Hosseini

Molecular Tectonics Laboratory

University of Strasbourg, CNRS

CMC UMR 7140, F-67000, France

E-mail: hosseini@unistra.fr

Keywords: Optical Waveguides, Molecular Crystals, Molecular Tectonics, Smart Materials, Optoelectronics

Molecular crystals are promising anisotropic optical transducing media for next-generation optoelectronic microdevices that will be capable of secure transduction of information and impervious to external electromagnetic interference. However, their full potential has not been explored yet due to their poor capability of processing and mechanical compliance; the pronounced brittleness and proneness for cracking that often result in irrecoverable damage. These issues are detrimental to their ability to transduce light. Here a novel strategy is presented 


\section{WILEY-VCH}

based on 3D epitaxial crystal growth of organic/inorganic crystals based on charge assisted Hbonds, that can be used to efficiently weld broken molecular single-crystalline optical waveguides, restoring their light-transducing capability. This approach can also be applied to prepare asymmetric multidomain crystalline heterostructures starting from isostructural molecular tectons, resulting in novel opto/electro/mechanical functionalities in the hybrid materials. It also removes an important obstacle towards wider application of molecular crystals in the next-generation optoelectronics.

\section{Introduction}

Molecular single crystals are rapidly emerging as building blocks for next-generation optoelectronics, and these prospects are based on their long-range structural order and anisotropy, tunability of their structures by small chemical variations, and costeffectiveness. ${ }^{[1-4]}$ These assets favor organic crystals for a broad range of applications, including light-emitting devices, ${ }^{[5]}$ organic field-effect transistors ${ }^{[6]}$ photodetectors, ${ }^{[7]}$ photovoltaic cells, ${ }^{[8]}$ thermochemiluminescent materials, ${ }^{[9]}$ solid-state lasers, ${ }^{[10]}$ and optical waveguides. ${ }^{[11-13]}$ This latter research direction is particularly prolific, given the increasing number of challenges for a secure transfer of information in a globally hyperconnected world. Compared to the traditionally used silica-based and polymeric materials, molecular crystals appear as viable candidates for future organic optical waveguides due to their high-refractive index $(n=1.5-1.8)$ required for efficient optical confinement. Other advantages, such as nearly defect-free structures, modular optical and electronic properties that can be altered by virtue of simple chemical modifications, and the possibility to control their morphology add further value to these underexplored materials. ${ }^{[14-19]}$ As some of the additional structural and functional flexibility they provide, the integration of photoactive components, such as $\pi$-conjugated molecular building blocks, can enable dual-mode light transduction, namely active and passive waveguiding, depending on the wavelength of the input light. ${ }^{[20]}$ Within this context, passive 


\section{WILEY-VCH}

light transduction implies transmission of the unaltered input light across the crystal, while active transduction refers to electronic excitation by the incident light and transduction of the crystal's own photoluminescence from the excitation spot to the edges of the crystal.

By using a combination of chemical and crystal engineering techniques, the waveguiding properties of molecular crystals can be combined with a broad range of other functionalities to prepare new multifunctional materials. Depending on the nature of the starting molecular components, hybrid single crystals can present a broad range of properties such as generation of chiro-optical signals, ${ }^{[21,22]}$ mechanical compliance, ${ }^{[19,20,23-26]}$ photocontrol over their optical

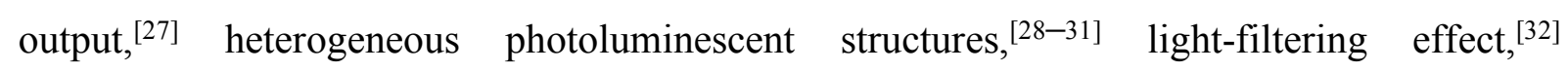
mechanofluorochromism, ${ }^{[33]}$ optical modulation, ${ }^{[34]}$ logic gate operations, ${ }^{[35-37]}$ field-effect optical waveguiding, ${ }^{[38]}$ and application of bio-based design. ${ }^{[39,40]}$ The virtually limitless possibilities that this class of materials offers for the development of novel advanced photonic devices necessitates further research into the drawbacks of these prototypical systems. Apart from a very few exceptions, ${ }^{[41-43]}$ ostensibly one of the most serious drawbacks of molecular crystals, among them organic crystals, is their brittleness, which results in difficulties with processing into transducing bodies of a desired length or coupling between crystals and also with other optoelectronic components. Transduction of light signals between multiple waveguides, a feature that is of paramount importance for fabrication of optical circuits, is currently only possible by mechanical coupling of different crystals in tip-to-tip or crossed geometries. Unfortunately, this leads to optical leakage and drastic decrease of light intensity due to poor coupling and elastic and inelastic light scattering. ${ }^{[4]}$

To overcome these limitations, we took advantage of the 3D epitaxial crystal growth to "weld" at room temperature and under mild conditions active molecular optical waveguides and to prepare compositionally "sequenced" modular crystals that not only provide alternative solution to the problem of crystal brittleness, but ultimately also extend the functionalities and the potential scope of application of single-crystal optical waveguides. To that end, here we 


\section{WILEY-VCH}

report the application of a supramolecular approach based on the molecular tectonics ${ }^{[45]}$ that allows hierarchical construction of periodic crystalline architectures at both nanoscale (molecular networks) ${ }^{[46]}$ and macroscale (crystalline networks). ${ }^{[47]}$ As it has been reported previously, ${ }^{[48-50]}$ by combining isomorphous and almost isometric (isostructural) crystalline architectures, it is possible to generate a vast series of multidomain core-shell, and welded molecular crystals under properly defined conditions. ${ }^{[51,52]}$

\section{Results and Discussion}

Specifically, we focused on two hydrogen-bonded molecular networks based on the hydrogen bond donor tecton 2,2'-(1,4-phenylene)-bisamidinium dication $\left(\mathbf{1}^{2+}\right)$ and $\left[\mathrm{M}^{\mathrm{II}}(\mathrm{CN})_{6}\right]^{4-}$, of formula $\mathbf{1}_{2}\left[\mathrm{M}^{\mathrm{II}}(\mathrm{CN})_{6}\right] \cdot 8 \mathrm{H}_{2} \mathrm{O}(\mathrm{M}=\mathrm{Ru}$ and $\mathrm{Fe})$, which are well-known to crystallize as isostructural crystalline compounds (compound $\mathbf{A}$ with $\mathrm{M}=\mathrm{Ru}$ and $\mathbf{B}$ with $\mathrm{M}=\mathrm{Fe}$ ). ${ }^{[53]}$ The resulting structure is based on a $2 \mathrm{D}$ supramolecular network formed by octahedral metal hexacyanide anions interconnected via $\mathbf{1}^{2+}$ through charge-assisted hydrogen bonds, as shown in Figure 1 (A: triclinic, $P \overline{1}, a=7.6658(2) \AA, b=10.9443(3) \AA, c=13.4958(4) \AA, \alpha=$ $70.252(2)^{\circ}, \beta=75.065(2)^{\circ}, \gamma=85.455(2)^{\circ}$ and $V=1029.63(5) \AA^{3}$; B: triclinic, $P \overline{1}, a=7.6538(2)$ $\AA, b=10.9276(3) \AA, c=13.4639(3) \AA, \alpha=70.260(5)^{\circ}, \beta=75.085(5)^{\circ}, \gamma=85.502(5)^{\circ}$ and $V$ $\left.=1024.16(4) \AA^{3}\right)$. The planar supramolecular architectures are connected through H-bonded water bridges, as previously reported (see SI Figure S4). ${ }^{[53]}$ As shown in Figure 2 and in the Supporting Information (SI) Section S1, by using a simple procedure, these isostructural compounds readily form core-shell and welded hierarchical crystalline architectures.

A can be easily crystallized as millimeter-long colorless rod-shape crystals from aqueous solution of $\mathbf{1}^{2+}$ and $\left[\mathrm{Ru}^{\mathrm{II}}(\mathrm{CN})_{6}\right]^{4-}$ in 2:1 molar ratio via slow evaporation at room temperature (more experimental details are provided in the SI). The crystals of both species have the fastest growing axis along the [100] direction and they grow with (001) as the widest face, see Figure 


\section{WILEY-VCH}

1B. The homowelded crystals were prepared by first cutting a seed single crystal of one compound, for example A, into two pieces (as shown in Figure 2A and 2B). The two resulting crystals were then disposed with the same spatial orientation relative to the (001) crystal face, aligned with a gap of $c a .100 \mu \mathrm{m}$ between them and covered with a solution containing the components for crystallization of $\mathbf{A}$, as shown in Figure 2A. In a first approximation, epitaxial growth of A occurred between both fragments of the seed crystal as well as at the two extremities of the welded crystal. The resulting welded crystal is composed of five crystalline zones with same chemical composition, A-A-A-A-A along the [100] direction (SI Section S1 for further experimental details). The heterowelded crystals were prepared by first cutting a seed single crystal of one compound, for example A, into two pieces (Figure 2C). As for homowelding, the two resulting crystals were aligned and then covered with a solution containing the components for crystallization of the isostructural compound B. Again, in a first approximation, the epitaxial growth of $\mathbf{B}$ occurred between both components of $\mathbf{A}$ as well as at the two extremities of the welded crystal. The resulting welded crystal is composed of five crystalline zones that differ by their composition, B-A-B-A-B along the [100] direction (see Figure 2C and SI Section S1 for further experimental details). The crystal sectors of $\mathbf{A}$ and $\mathbf{B}$ can be distinguished by their colour.

Inspired by the compatibility of A and $\mathbf{B}$ for epitaxial growth, ${ }^{[54]}$ we prepared "restricted" coreshell crystals: usually the A@B core-shell crystals present the ABA composition, as shown in Figures S1 and S2 of the SI, while in the case of a "restricted" core-shell crystal, only the AB composition is observed, Figures 2D and 2E. This has been obtained using a mineral gel, that exposes one face of the seed crystal for further growth, as depicted in Figure 2D and in the SI Section $1 .^{[55]}$

When crystals of A are exposed to $365 \mathrm{~nm}$ UV light (LED), they emit orange light with a broad emission profile having a maximum centered around $500 \mathrm{~nm}$ (Figure 3). The pronounced photoluminescence possibly originates from the second-sphere charge-transfer transition 


\section{WILEY-VCH}

between the ionic pair. ${ }^{[56]}$ When the UV light is focused on one end of the crystal, the photoluminescence is transduced throughout the crystal to the opposite terminus as a result of efficient optical confinement within the crystalline lattice (Figure 3C). To further test the efficiency of $\mathbf{A}$ as active optical waveguide, the optical loss coefficient ( $\left.\alpha^{\prime}\right)$ was measured of a representative crystal with length of $2.6 \mathrm{~mm}$. The crystal was excited with a $365 \mathrm{~nm}$ focused LED light at four different locations perpendicular to its long axis, and the relative photoluminescence intensity maps were recorded, while filtering the input UV light with a 450 nm long-pass filter (see SI Section S3 for details). The outcoupling efficiency was obtained by analyzing the dependence of the output light intensity $\left(I_{\text {tip }}\right)$ over the intensity at the excitation point ( lbody $_{\text {) }}$ as function of the optical path (Figure 3D; SI Section S4) using the single exponential fitting equation $I_{\text {tip }} / I_{\text {body }}=\mathrm{e}^{-\alpha d}$, where $\alpha$ is the optical loss expressed in $\mathrm{mm}^{-1}$ and $d$ is the distance travelled by the light within the crystal. We then expressed $\alpha$ in $\mathrm{dB} \mathrm{mm}^{-1}$ by using the relation $\alpha^{\prime} /\left(\mathrm{dB} \mathrm{mm}^{-1}\right) \approx 4.34 \alpha / \mathrm{mm}^{-1}$ (see SI Section S4 for further details). The value of $\alpha$ ' was found to be $0.8 \pm 0.3 \mathrm{~dB} \mathrm{~mm}^{-1}$, and is close to the best-performing organic singlecrystalline waveguides of similar size reported to date. ${ }^{[57]}$ Considering the negligible overlap between absorption and emission bands of $\mathbf{A}$, the optical loss can be attributed to surface roughness and defects. ${ }^{[58]}$

In order to test the efficacy of welded crystals as optical waveguides, a series of homowelded crystals of A-A-A-A-A composition were prepared, as described above (Figure 2; SI Section S1), and their performance in transduction of light was compared to that of mechanically coupled A crystals. Both welded and coupled crystals were excited at one end and their photoluminescence intensity maps were recorded. The light intensity was integrated over the length of such waveguides (Figure 4, panels A and B). This procedure was repeated for three different welded crystals and three coupled crystals of $\mathbf{A}$. The welded crystals consistently showed higher outcoupling efficiency with the inevitable light leakage at the welding/joint interface due to the presence of crystal defects that were generated during crystallization (Figure 


\section{WILEY-VCH}

2; SI Section S1). This result indicates that the welding process does not have a significant effect on decreasing the optical losses at the joint interface. The epitaxial growth allows for high degree of the partial restoration of the crystalline lattice, thereby leading to more efficient optical confinement and light coupling compared to the axially coupled crystals. While heterowelded crystals, as the ones shown in Figure 2, are conceptually interesting systems, we decided to explore the light transduction properties of structurally anisotropic heterostructures to explore novel functionalities and with this purpose we focused our attention on crystals possessing AB composition.

The A@B "restricted" core-shell crystal was analyzed as long-pass filter for white light (Figure 5). ${ }^{[59]}$ We first confirmed that pure single crystals of $\mathbf{A}$, taken as reference, work as passive waveguides to LED light. Then we performed the same measurement with the A@B "restricted" crystal and we compared the output spectra from the reference and the "restricted" crystal. The output spectrum of $\mathbf{A} @ \mathbf{B}$ showed an optical output intensity relative decrease of nearly $90 \%$ of the blue component of the white LED light at $460 \mathrm{~nm}$ compared to pure $\mathbf{A}$ as evidence of its efficiency to absorb light $<580 \mathrm{~nm}$, given the absorption spectrum of $\mathbf{B}$ (Figure 5B).

To assess the passive waveguiding capability of the sequenced crystal, we tested the light transduction of light with wavelengths outside the absorption windows of both $\mathbf{A}$ and $\mathbf{B}$. We used laser diodes as input light sources with wavelengths in the near IR (660 nm), commonly used in plastic optical fibers light transmission, and in the IR (1310 and $1550 \mathrm{~nm})$, that are used in glass-based fiber optics (SI Section 3). As expected, the signals were transduced unaltered through A@B (Figure 5C), and this result is promising for integration of this prototypical material in commercial optical devices.

\section{Conclusion}




\section{WILEY-VCH}

These results clearly indicate that 3D epitaxial growth can be used as an effective strategy to repair and/or extend split single-crystalline optical waveguides and to create multifunctional heterostructures, which can act both as light-transducing media and as long-pass filters at room temperature and under mild conditions. Specifically, we have demonstrated that broken or otherwise damaged crystalline waveguides can be efficiently welded under mild conditions, and this procedure can partially restore their light outcoupling efficiency. It is worth mentioning that the crystal growth of welded crystals in nonconfined space is not restricted to one direction, but it extends to all three dimensions, as previously reported. ${ }^{[51]}$ The preferential crystal growth along a specific direction is accompanied by the formation of shell structure in the other directions. To obtain 1D crystal growth studies in confined media are ongoing and they will be published elsewhere. ${ }^{[55]}$ We also used 3D epitaxy to obtain a "restricted" core-shell crystal. We successfully applied these asymmetric sequenced molecular crystals as optical waveguides with long-pass filter incorporated in the crystalline lattice. These prototypical optoelectronic devices showed passive waveguiding properties in the telecommunications radiation range. This approach introduces a way to increase the workability and post-processing of molecular single crystals as optical waveguides towards novel and unexplored applications, opening new avenues in the design and synthesis of future optoelectronics based on molecular single crystals.

\section{Supporting Information}

Supporting Information is available from the Wiley Online Library or from the author.

\section{Acknowledgements}

We thank New York University Abu Dhabi for the financial support for this work. This research was partially carried out using the Core Technology Platform resources at New York University Abu Dhabi. We also thank the University of Strasbourg, the C.N.R.S., the International centre for Frontier Research in Chemistry (icFRC), the Labex CSC within the Investissement d'Avenir 


\section{WILEY-VCH}

program ANR-10-IDEX-0002-02 (Ph. D. fellowship to J. B.) and the Ministère de l'Enseignement Supérieur, de la Recherche et de l'Innovation.

Received: ((will be filled in by the editorial staff))

Revised: ((will be filled in by the editorial staff))

Published online: ((will be filled in by the editorial staff))

References and Notes

[1] Y. Wang, L. Sun, C. Wang, F. Yang, X. Ren, X. Zhang, H. Dong, W. Hu, Chem. Soc. Rev. 2019, 48, 1492-1530.

[2] Y. Huang, Z. Wang, Z. Chen, Q. Zhang, Angew. Chem. Int. Ed. 2019, 58, 9696-9711.

[3] X. Zhang, H. Dong, W. Hu, Adv. Mater. 2018, 30, 1801048.

[4] E. Ahmed, D. P. Karothu, P. Naumov, Angew. Chem. Int. Ed. 2018, 57, 8837-8846.

[5] R. Ding, F.-X. Dong, M.-H. An, X.-P. Wang, M.-R. Wang, X.-B. Li, J. Feng, H.-B. Sun, Adv. Funct. Mater. 2019, 29, 1807606.

[6] A. Yamamura, S. Watanabe, M. Uno, M. Mitani, C. Mitsui, J. Tsurumi, N. Isahaya, Y. Kanaoka, T. Okamoto, J. Takeya, Science Advances 2018, 4, eaao5758.

[7] D. Periyanagounder, T.-C. Wei, T.-Y. Li, C.-H. Lin, T. Piechota Gonçalves, H.-C. Fu, D.-S. Tsai, J.-J. Ke, H.-W. Kuo, K.-W. Huang, N. Lu, X. Fang, J.-H. He, Adv. Mater. 2020, 32, 1904634.

[8] M. Kikuchi, S. Makmuang, S. Izawa, K. Wongravee, M. Hiramoto, Org. Electron. 2019, $64,92-96$.

[9] S. Schramm, D. P. Karothu, N. M. Lui, P. Commins, E. Ahmed, L. Catalano, L. Li, J. Weston, T. Moriwaki, K. M. Solntsev, P. Naumov, Nat. Commun. 2019, 10, 997.

[10] G.-Q. Wei, X.-D. Wang, L.-S. Liao, Adv. Funct. Mater. 2019, 29, 1902981.

[11] C. Zhang, Y. S. Zhao, J. Yiao, Phys. Chem. Chem. Phys. 2011, 13, 9060-9073. 


\section{WILEY-VCH}

[12] R. Chandrasekar, Phys. Chem. Chem. Phys. 2014, 16, 7173-7183.

[13] H. Jiang, W. Hu, Angew. Chem. Int. Ed. 2020, 59, 1408-1428.

[14] H. Yanagi, T. Morikawa, Appl. Phys. Lett. 1999, 75, 187-189.

[15] Q. Bao, B. M. Goh, B. Yan, T. Yu, Z. Shen, K. P. Loh, Adv. Matter. 2010, 22, 36613666.

[16] W. Zhu, R. Zheng, Y. Zhen, Z. Yu, H. Dong, H. Fu, Q. Shi, W. Hu, J. Am. Chem. Soc. 2015, 137, 34, 11038-11046.

[17] N. Chandrasekhar, R. Chandrasekar, Angew. Chem. Int. Ed. 2019, 51, 3556-3561.

[18] S. Hayashi, T. Koizumi, Chem. Eur. J. 2018, 24, 8507-8512.

[19] R. Huang, C. Wang, Y. Wang, H. Zhang, Adv. Mater. 2018, 30, 1800814.

[20] L. Catalano, D. P. Karothu, S. Schramm, E. Ahmed, R. Rezgui, T. J. Barber, A. Famulari, P. Naumov, Angew. Chem. Int. Ed. 2018, 57, 17254-17258.

[21] D. Venkatakrishnarao, C. Sahoo, E. A. Mamonov, V. B. Novikov, N. V. Mitetelo, S. R. G. Naraharisetty, T. V. Murzina and R. Chandrasekar, J. Mater. Chem. C 2017, 5, 12349-12353. [22] N. Mitetelo, D. Venkatakrishnarao, J. Ravi, M. Popov, E. Mamonov, T. V. Murzina, R. Chandrasekar, Adv. Opt. Mater. 2019, 7, 1801775.

[23] H. Liu, Z. Lu, Z. Zhang, Y. Wang, H. Zhang, Angew. Chem. Int. Ed. 2018, 57, 84488452.

[24] B. Liu, Q. Di, W. Liu, C. Wang, Y. Wang, H. Zhang. J. Phys. Chem. Lett. 2019, 10, $1437-1442$.

[25] H. Liu, Z. Bian, Q. Cheng, L. Lan, Y. Wang, H. Zhang, Chem. Sci. 2019, 10, 227-232.

[26] R. Huang, B. Tang, K. Ye, C. Wang, H. Zhang, Adv. Optical Mater. 2019, 7, 1900927.

[27] J. Mahmoud Halabi, E. Ahmed, L. Catalano, D. P. Karothu, R. Rezgui, P. Naumov, J. Am. Chem. Soc. 2019, 141, 14966-14970.

[28] Z.-Z. Li, Y.-C. Tao, X.-D. Wang, L.-S. Liao, ACS Photonics, 2018, 5, 3763-3771. 


\section{WILEY-VCH}

[29] L. Catalano, P. Commins, S. Schramm, D. P. Karothu, R. Rezgui, K. Hadef, P. Naumov, Chem. Commun. 2019, 55, 4921-4924.

[30] M.-P. Zhuo, J.-J. Wu, X.-D. Wang, Y.-C. Tao, Y. Yuan, L.-S. Liao, Nat. Commun. 2019, $10,3839$.

[31] V. V. Pradeep, M. Annadhasan, R. Chandrasekhar, Chem. Asian J. 2019, 14, 4577-4581.

[32] N. Chandrasekhar, S. Basak, M. A. Mohiddon, R. Chandrasekar, ACS Appl. Mater. Interfaces. 2014, 6, 1488-1494.

[33] S. Hayashi, S.-y. Yamamoto, D. Takeuchi, Y. Ie, K. Takagi, Angew. Chem. Int. Ed. 2018, 57, 17002-17008.

[34] D. Venkatakrishnarao, M. A. Mohiddon, N. Chandrasekhar, R. Chandrasekar, Adv. Opt. Mater. 2015, 3, 1035-1040.

[35] M.-P. Zhuo, Y.-C. Tao, X.-D. Wang, Y. Wu, S. Chen, L.-S. Liao, Angew. Chem. Int. Ed. 2018, 57, 11300-11304.

[36] Z.-Z. Li, J.-J. Wu, X.-D. Wang, K.-L. Wang, S. Zhang, W.-F. Xie, L.-S. Liao, Adv. Opt. Mater. 2019, 7, 1900373.

[37] X. T. Liu, K. Wang, Z. Chang, T.-H. Zhang, J. Xu, Y. S. Zhao, X.-H. Bu, Angew. Chem. Int. Ed. 2019, 58, 13890-13896.

[38] G. Zhao, H. Dong, Q. Liao, J. Jiang, Y. Luo, H. Fu, W. Hu, Nat. Commun. 2019, 10, 4790.

[39] B. Apter, N. Lapshina, A. Handelman, B. D. Fainberg, G. Rosenman, Small. 2019, 14, 1801147.

[40] W. Jin, B. Xue, Z. A. Arnon, H. Yuan, S. Bera, Q. Li, D. Zaguri, N. P. Reynolds, H. Li, Y. Chen, S. Gilead, S. Rencus-Lazar, J. Li, R. Yang, E. Gazit, ACS Nano. 2019, 13, $14477-$ 14485.

[41] P. Commins, H. Hara, P. Naumov, Angew. Chem. Int. Ed. 2016, 55, 13028-13032. 


\section{WILEY-VCH}

[42] P. Gupta, D. P. Karothu, E. Ahmed, P. Naumov, N. K. Nath, Angew. Chem. Int. Ed. 2018, 57, 8498-8502.

[43] E. Ahmed, D. P. Karothu, P. Naumov, Nat. Commun. 2019, 10, 3550.

[44] N. Chandrasekhar, M. A. Mohiddon, R. Chandrasekar, Adv. Opt. Mater. 2013, 1, 305311.

[45] M. W. Hosseini, Acc. Chem. Res. 2005, 4, 313-323.

[46] J. D. Wuest, Chem. Commun. 2005, 5830-5837.

[47] M. W. Hosseini, Chem. Commun. 2005, 5825-5829.

[48] S. Ferlay, M. W. Hosseini, Chem. Commun. 2004, 788-789.

[49] G. Marinescu, S. Ferlay, N. Kyritsakas, M. W. Hosseini, Chem. Commun. 2013, 1120911211.

[50] F. Zhang, C. R. R. Adolf, N. Zigon, S. Ferlay, N. Kyritsakas, M. W. Hosseini, Chem. Commun., 2017, 53, 3587-3590.

[51] C. R. R. Adolf, S. Ferlay, N. Kyritsakas, M. W. Hosseini, J. Am. Chem. Soc. 2015, 137, $15390-15393$.

[52] C. R. R. Adolf, S. Ferlay, M. W. Hosseini, CrystEngComm. 2018, 20, 2233-2236.

[53] S. Ferlay, V. Bulach, O. Felix, M. W. Hosseini, J.-M. Planeix, N. Kyritsakas, CrystEngComm, 2002, 4, 447-453.

[54] B. K. Olmsted, S. Ferlay, P. Dechambenoit, M.W. Hosseini, M. D. Ward, Cryst. Growth Des., 2009, 6, 2841-2847.

[55] C. R. Adolf, S. Ferlay, M. W. Hosseini, manuscript in preparation.

[56] V. Balzani, N. Sabbatini, F. Scandola, Chem. Rev. 1986, 86, 319-337.

[57] S. Min, A. Dhamsaniya, L. Zhang, G. Hou, Z. Huang, K. Pambhar, A. K. Shah, V. P. Mehta, Z. Liu, B. Song, J. Phys. Chem. Lett. 2019, 10, 5997-6002.

[58] N. Chandrasekhar, E. R. Reddy, M. D. Prasad, M. S. Rajadurai, R. Chandrasekar, CrystEngComm. 2014, 16, 4696-4700. 


\section{WILEY-VCH}

[59] A long-pass filter is an optical filter that attenuates shorter wavelengths and transmits longer wavelengths over a target range of the light spectrum.

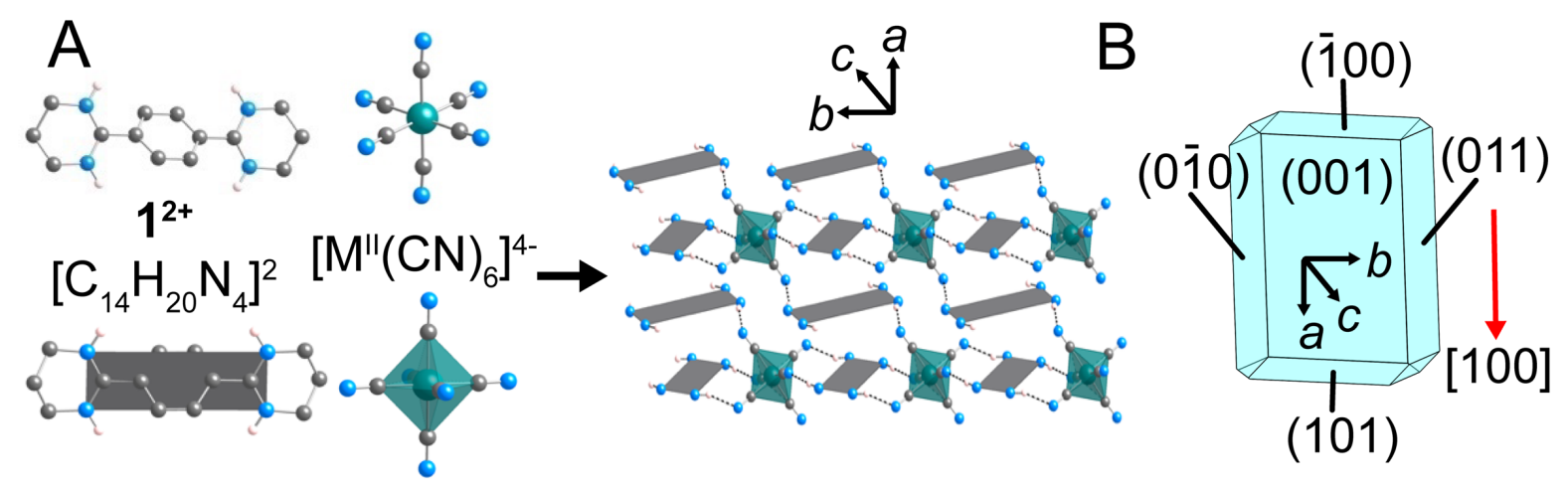

Figure 1. (A) Structure of the building blocks in the structures of $\mathbf{A}$ and $\mathbf{B}$ with H-bond highlighted with broken black lines. Water molecules are omitted for the sake of clarity. (B) The calculated BFDH crystal morphology of the two isostructural compounds. The fast crystal growth axis is highlighted with a red arrow. Atoms Color Code: C, grey; N, Blue; M (Fe or Ru), aquamarine; $\mathrm{H}$, White. 


\section{WILEY-VCH}

A

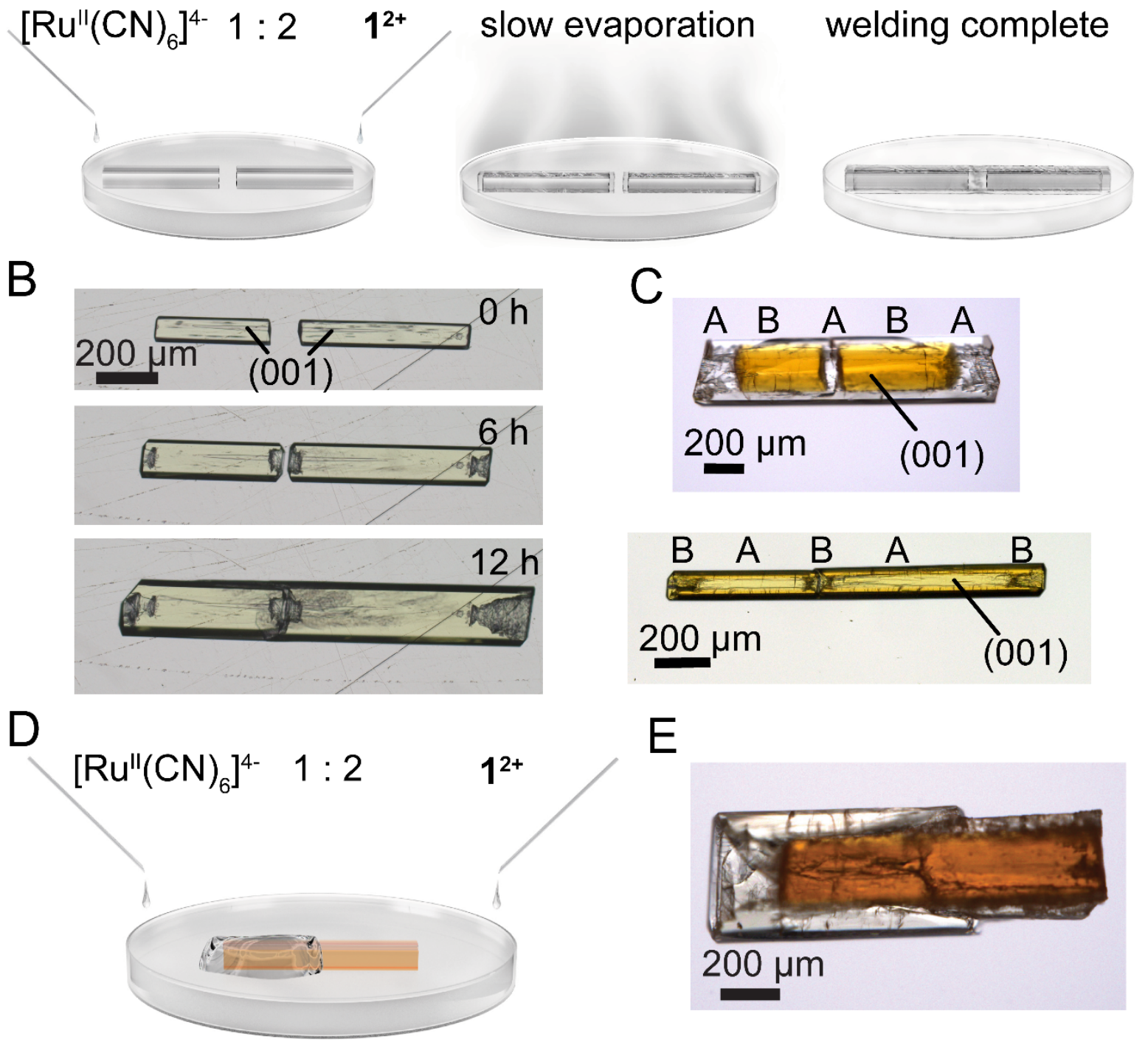

Figure 2. (A) Schematic representation of the homowelding. From left to right, the cartoon shows alignment of the broken crystal of $\mathbf{A}$, addition of precursors of $\mathbf{A}$, slow evaporation, and completion of the welding; (B) Homowelding of two fragments of a broken crystal of A leading to a single crystal with composition A-A-A-A-A; (C) Representative heterowelded crystals having A-B-A-B-A (top) and B-A-B-A-B (bottom) sequences with the (001) face highlighted. (D) Method for the preparation of the "restricted" core-shell crystal. (E) Optical image of the “restricted" core-shell crystal A@B. 


\section{WILEY-VCH}

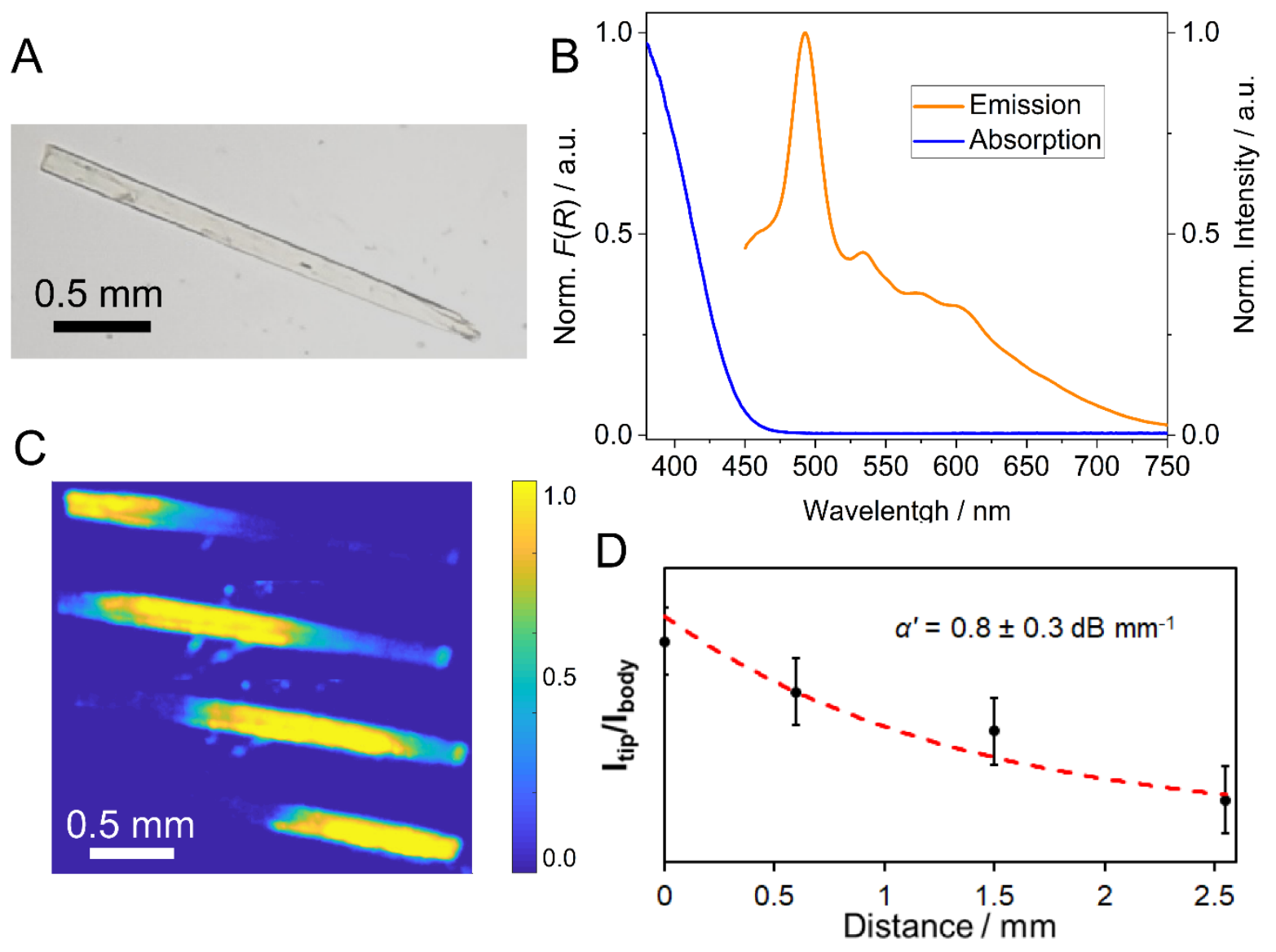

Figure 3. Optical waveguiding properties of $\mathbf{1}_{2}-\left[\mathrm{Ru}(\mathrm{CN})_{6}\right]$. (A) Representative needle-like crystal of A. (B) Normalized Kulbelka-Munk $(F(R))$ function and emission spectra of A. (C) Photoluminescence intensity maps of the crystal showed in panel A recorded with a $400 \mathrm{~nm}$ long-pass filter to remove the $365 \mathrm{~nm}$ excitation. (D) Single-exponential fit of the decreasing $I_{\text {tip }} / I_{\text {body }}$ with increasing tip-to-excitation distance plotted for the photoluminescence of $\mathbf{A}$. 


\section{WILEY-VCH}
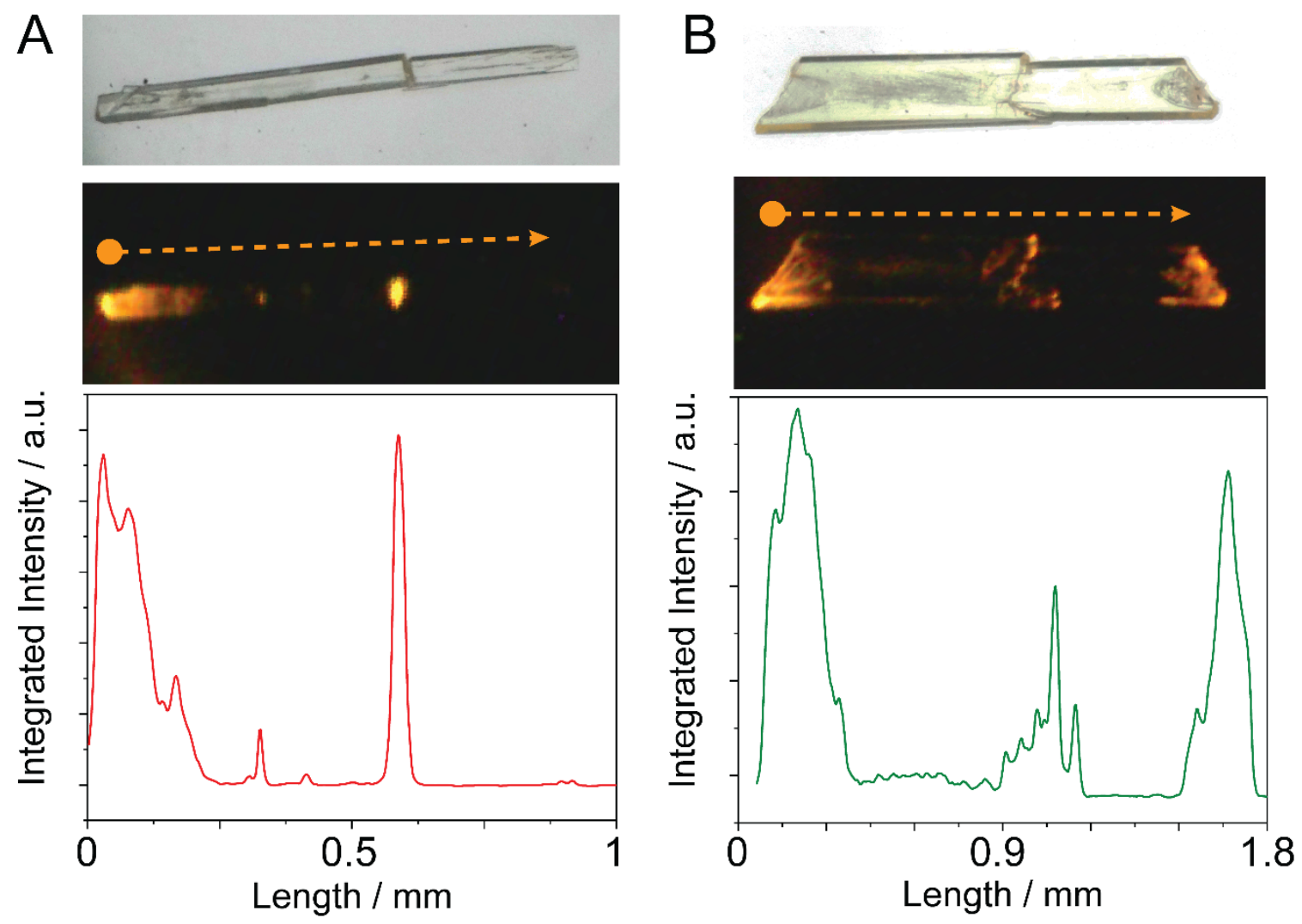

Figure 4. (A) Light transduction through two fragments of a broken crystal of $\mathbf{A}$ that were mechanically coupled with each other. Shown are the two fragments under visible light (top), dark-field image of the photoluminescence recorded by using a $450 \mathrm{~nm}$ long-pass filter (middle), and integrated intensity of the photoluminescence maps (bottom). The orange broken line indicates the direction of light propagation. (B) Waveguiding performance of two welded crystals of A. Shown are the welded crystal under visible light (top), dark-field image of the photoluminescence recorded by using a $450 \mathrm{~nm}$ long-pass filter (middle), and integrated intensity of the photoluminescence maps (bottom). The orange broken line indicates the direction of light propagation. 
WILEY-VCH

A

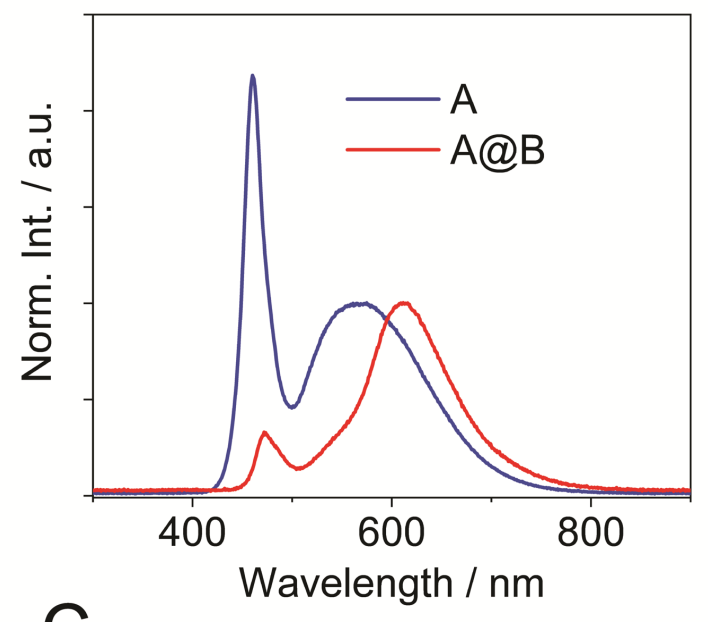

C

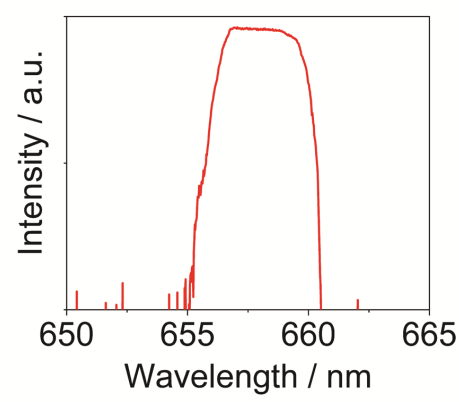

B
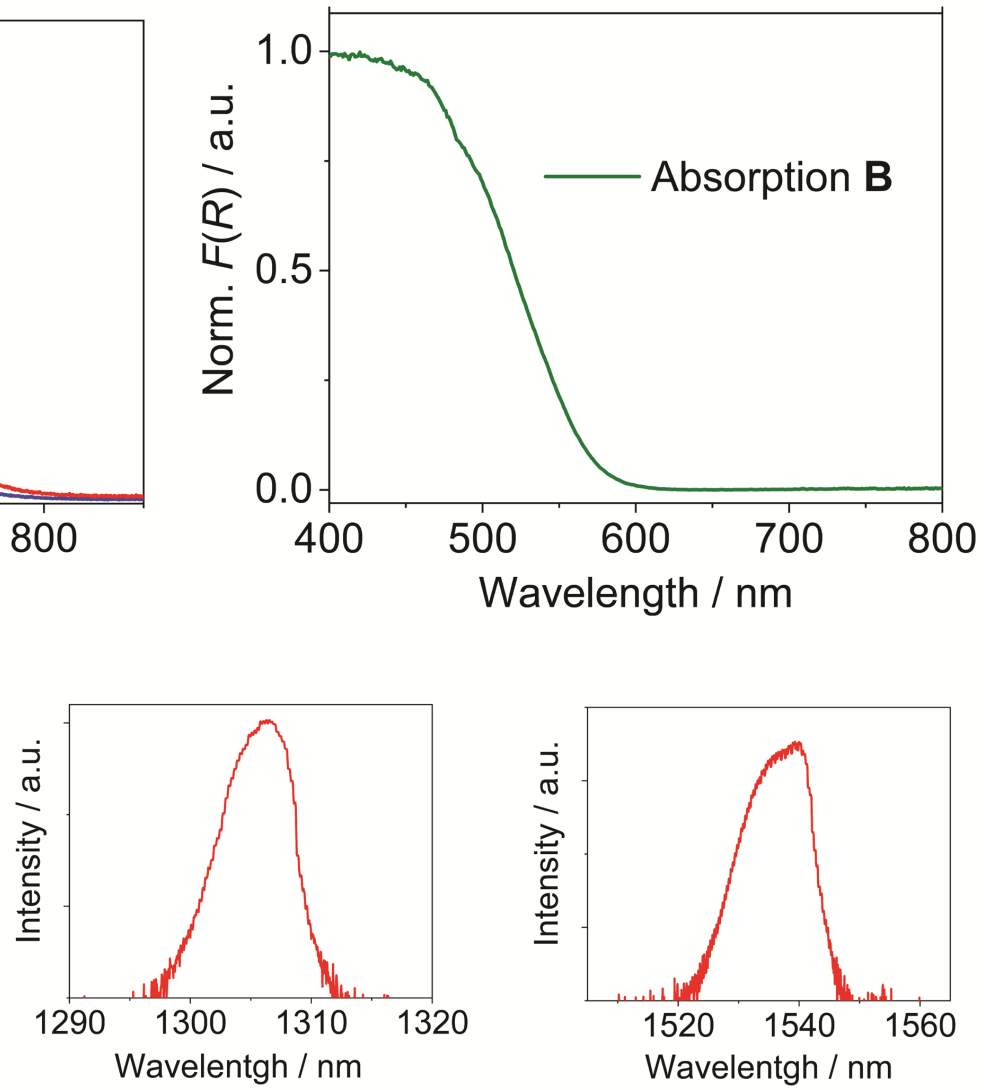

Figure 5. (A) Optical output from pure A and A@B using white light as input. (B) Normalized $F(R)$ function of pure B. (C) Near IR and telecom IR output light signals passively transduced through A@B. 


\section{WILEY-VCH}

A simple strategy based on 3D epitaxial crystal growth was devised to weld and repair broken single-crystalline optical waveguides, restoring their light-transduction capability. The same approach can be used to build asymmetric multidomain heterostructures, which can introduce novel functionalities to the final materials towards next-generation optoelectronics.

Keyword Advanced Functional Materials

L. Catalano, J. Berthaud, G. Dushaq, D. P. Karothu, R. Rezgui, M. Rasras, S. Ferlay, M. W. Hosseini, * P. Naumov*

Sequencing and Welding of Molecular Single-Crystal Optical Waveguides

crystalline optical waveguide broken welded 